\title{
SUBSPACE-HYPERCYCLIC TUPLES OF OPERATORS
}

\author{
Mansooreh Moosapoor \\ Farhangian University \\ Postcode 4166616711, Rasht, IRAN
}

\begin{abstract}
In this paper we introduce subspace-hypercyclic tuples of operators and construct interesting examples of such operators. We state some sufficient conditions for $\mathrm{n}$-tuples of operators to be subspace-hypercyclic. Surprisingly, we prove that subspace-hypercyclic tuples exist on finite-dimensional spaces.
\end{abstract}

AMS Subject Classification: 47A16, 47B37, 37B99

Key Words: hypercyclic operators, hypercyclic tuples, subspace-hypercyclic tuples

\section{Introduction}

Let $X$ be a Banach space. An operator $T$ on $X$ is called hypercyclic, if there exists a vector $x \in X$ whose orbit under $T$, $\operatorname{orb}(T, x)=\left\{x, T x, T^{2} x, \ldots\right\}$, is dense in $X$. We call $x$ a hypercyclic vector for $T$.

A good reference about hypercyclicity is the book of Grosse-Erdmann and Peris [5]. For more information about this concept one can also see [2] and [4].

By an $n$-tuple of operators we mean a finite sequence of length $n$ of commuting continuous linear operators on a Banach space $X$.

Definition 1.1. Let $T=\left(T_{1}, T_{2}, \ldots, T_{n}\right)$ be an $n$-tuple of operators acting on an infinite dimensional Banach space $X$ and let

$$
F=F_{T}=\left\{T_{1}^{k_{1}} T_{2}^{k_{2}} \ldots T_{n}^{k_{n}}: k_{i} \geq 0, i=1, \ldots, n\right\},
$$

be the semigroup generated by $T$. For $x \in X$, the orbit of $x$ under the tuple $T$

Received: November 28, 2015

Published: March 23, 2016
(C) 2016 Academic Publications, Ltd.

url: www.acadpubl.eu 
is

$$
\operatorname{orb}(T, x)=\{S x: S \in F\} .
$$

A vector $x \in X$ is called a hypercyclic vector for $T$, if $\operatorname{orb}(T, x)$ is dense in $X$. In this case the tuple $T$ is called hypercyclic. The set of all hypercyclic vectors of $T$ is denoted by $H C(T)$.

Feldmann in [3] proved some interesting properties of these operators. Also in [12] one can find more results about hypercyclic tuples. Recently, Madore and Martinez-Avendano in [7] introduced the concept of subspace-hypercyclicity for an operator as follows.

Definition 1.2. Let $T$ be a bounded linear operator on $X$ and let $M$ be a nonzero closed subspace of $X$. We say that $T$ is subspace-hypercyclic for $M$ if there exists $x \in X$ such that $\operatorname{orb}(T, x) \cap M$ is dense in $M$. We call $x$ a subspace-hypercyclic vector for $T$.

For example let $T$ be a bounded linear operator on $X$ and let $I$ be the identity operator on $X$. Then $T \oplus I: X \oplus X \rightarrow X \oplus X$ is subspace-hypercyclic for the subspace $M:=X \oplus\{0\}$ with subspace-hypercyclic vector $x \oplus 0$. Clearly, $T \oplus I$ is not hypercyclic.

Rezai in [10] answered some questions of Madore and Avendano asked in [7]. One can also see [6], [8] and [9] for more information.

This paper is organized as follows. In Section 2 we introduce subspacehypercyclic tuples of operators and we give some interesting examples of such operators. In Section 3 we show that surprisingly subspace-hypercyclic tuples exist on finite-dimensional spaces.

Throughout this paper, we assume that $B(X)$ is the space of bounded linear operators acting on a separable and infinite dimensional complex Banach space $X$. We also denote by $M$ a nonzero closed subspace of $X$.

\section{Definitions and Examples}

We first introduce the notion of a subspace-hypercyclic tuple with respect to a non-zero closed subspace $M$ of $X$ as follows.

Definition 2.1. Let $T=\left(T_{1}, T_{2}, \ldots, T_{n}\right)$ be an $n$-tuple of operators acting on $X$ and let

$$
F=F_{T}=\left\{T_{1}^{k_{1}} T_{2}^{k_{2}} \ldots T_{n}^{k_{n}}: k_{i} \geq 0, i=1, \ldots, n\right\},
$$

be the semigroup generated by $T$. For $x \in X$, the orbit of $x$ under $T$ is the set

$$
\operatorname{orb}(T, x)=\{S x: S \in F\} .
$$


A vector $x \in X$ is called an $M$-hypercyclic vector for $T$ if $\operatorname{orb}(T, x) \cap M$ is dense in $M$. In this case we say that $T$ is a subspace-hypercyclic tuple with respect to $M$ or an $M$-hypercyclic tuple.

The set of all $M$-hypercyclic vectors for $T$ is denoted by $H C(T, M)$.

Theorem 2.2. Let $T=\left(T_{1}, T_{2}, \ldots, T_{n}\right)$ be an $n$-tuple of operators acting on $X$. If the semigroup $F_{T}$ contains an $M$-hypercyclic operator, then $T$ is a subspace-hypercyclic tuple with respect to $M$.

Proof. Let $S \in F_{T}$ be an $M$-hypercyclic operator with a hypercyclic vector $x$. Then,

$$
M=\operatorname{cl}(\operatorname{orb}(S, x) \cap M) \subseteq \operatorname{cl}(\operatorname{orb}(T, x) \cap M) \subseteq M .
$$

Hence $\operatorname{cl}(\operatorname{orb}(T, x) \cap M)=M$ and $T$ is an $M$-hypercyclic tuple.

We first give a simple example of a subspace-hypercyclic tuple.

Example 2.3. Let $A$ and $B$ be hypercyclic operators on $X$ and let $I$ be the identity operator on $X$. Let $T_{1}=A \oplus I, T_{2}=I \oplus B$ and $T_{3}=I \oplus C$ where $C$ is an arbitrary operator on $X$. Then $T=\left(T_{1}, T_{2}, T_{3}\right)$ is a subspace-hypercyclic tuple with respect to $X \oplus\{0\}$ and $\{0\} \oplus X$, since $T_{1}$ is subspace-hypercyclic with respect to $X \oplus\{0\}$ and $T_{2}$ is subspace-hypercyclic with respect to $\{0\} \oplus X$.

Using Example 2.3, one can obtain different subspace-hypercyclic tuples. Recall that on $l^{2}$, the Hilbert space of all square summable complex sequences, the backward shift $B$ is defined as

$$
B\left(x_{0}, x_{1}, x_{3}, \ldots\right)=\left(x_{1}, x_{2}, \ldots\right) .
$$

It was shown in [11] that if $\lambda$ is a scalar with $|\lambda|>1$, then $\lambda B$ is a hypercyclic operator. By using this fact, we give an example of a subspace-hypercyclic tuple that is not a hypercyclic tuple as follows.

Example 2.4. Let $T=(I \oplus 2 B, I \oplus 3 B)$, where $I$ is the identity operator and $B$ is the backward shift on $l^{2}$. Let $x$ be a hypercyclic vector for $2 B$. Then $0 \oplus x$ is a subspace-hypercyclic vector for the tuple $T$ with respect to subspace $M:=\{0\} \oplus l^{2}$, since

$$
c l(\operatorname{orb}(T,(0 \oplus x)) \cap M)=\{0\} \oplus l^{2} .
$$

Also it is clear that $T$ is not a hypercyclic tuple. 
Example 2.5. Let $\lambda$ be a scalar with $|\lambda|>1$ and let $B$ be the backward shift on $l^{2}$. Let $m \in N$ and let

$$
M:=\left\{\left\{a_{n}\right\}_{n=0}^{\infty}: a_{n}=0 \quad \text { for } \quad n<m\right\} .
$$

Define $T=(I, \lambda B)$. Note that $\lambda B$ is an $M$-hypercyclic operator by Example 3.8 in [7]. So, $T$ is an $M$-hypercyclic tuple by Theorem 2.2.

Example 2.6. Let $B$ be the backward shift on $l^{2}$ and let $\lambda$ be a scalar with $|\lambda|>1$. By Example 3.7 in [7], $\lambda B$ is $M$-hypercyclic with respect to

$$
M:=\left\{\left\{a_{n}\right\}_{n=0}^{\infty}: a_{2 k}=0 \text { for all } k\right\} .
$$

Define $T=\left(\lambda B, \frac{1}{\lambda} B\right)$. Note that $\lambda B \in F_{T}$ and it is an $M$-hypercyclic operator. So $T$ is an $M$-hypercyclic tuple by Theorem 2.2 .

\section{Finite Dimensions}

Subspace-hypercyclic operators does not exist on finite dimensional spaces. Also it is proved in [7] that an operator can not be subspace-hypercyclic with respect to a finite-dimensional subspace . But surprisingly, we prove that subspacehypercyclic tuples exist on finite-dimensional spaces.

Theorem 3.1. (see [7]) Let $H$ be a finite-dimensional Hilbert space. If $T \in B(H)$, then $T$ is not subspace-hypercyclic for any closed nonzero subspace $M$ of $H$.

Theorem 3.2. (see [7]) Let $T \in B(H)$ where $H$ is a finite-dimensional Hilbert space. If $T$ is subspace-hypercyclic for a subspace $M$, then $M$ is not finite-dimensional.

Theorem 3.3. (see [3]) If $a, b>1$ are relatively prime integers, then $\left\{\frac{a^{n}}{b^{k}}: n, k \in \mathbb{N}\right\}$ is dense in $\mathbb{R}^{+}$, the positive real numbers.

In the following example we show that subspace-hypercyclic tuples can exist on finite-dimensional spaces.

Example 3.4. Let $I$ be the identity operator on the real Hilbert space $\mathbb{R}$ and let $T=\left(-2 I \oplus I, \frac{1}{3} I \oplus I\right)$ be a tuple on finite-dimensional space $\mathbb{R} \oplus \mathbb{R}$. By Theorem 3.3, $\operatorname{cl}(\operatorname{orb}(T,(1 \oplus 0)) \cap M)=M$. Then $T$ is a subspace-hypercyclic tuple with respect to finite-dimensional subspace $M:=\mathbb{R} \oplus\{0\}$. Note that $T$ is not a hypercyclic tuple on $\mathbb{R} \oplus \mathbb{R}$.

By generalizing previous example, we get the following theorem: 
Theorem 3.5. There are subspace-hypercyclic tuples of operators on $\mathbb{R}^{n}$ for any integer $n$ greater than 1.

Proof. Suppose that $a, b>1$ are relatively prime integers. Fix $a, b$ and consider $T=\left(-|a| I_{\mathbb{R}} \oplus I_{\mathbb{R}} \oplus \ldots \oplus I_{\mathbb{R}}, \frac{1}{|b|} I_{\mathbb{R}} \oplus I_{\mathbb{R}} \oplus \ldots \oplus I_{\mathbb{R}}\right)$ as an $n$-tuple on $\mathbb{R}^{n}=\mathbb{R} \oplus \ldots \oplus \mathbb{R}, n$ times. Then, $T$ is a subspace-hypercyclic $n$-tuple with respect to $M:=\mathbb{R} \oplus\{0\} \ldots \oplus\{0\}$, with $M$-hypercyclic vector $1 \oplus 0 \oplus \ldots \oplus 0$.

Note that the above theorem shows that, unlike the subspace-hypercyclic operators, subspace-hypercyclic tuples exist on finite-dimensional spaces.

Recall that if $T=\left(T_{1}, T_{2}\right)$ is a commuting pair of operators and $n=\left(n_{1}, n_{2}\right)$ is a pair of nonnegative integers (a multi-index), then we define $T^{n}$ to be the pair $\left(T^{n_{1}}, T^{n_{2}}\right)$. Ansari in [1] showed that if $T$ is a hypercyclic operator, then $T^{n}$ is also hypercyclic for any positive integer $n$. Feldmann in [3] showed that if $T$ is a hypercyclic tuple, then $T^{n}$ may not be hypercyclic for a multi-index $n=\left(n_{1}, n_{2}\right)$. So, it is natural to ask that if $T$ is a subspace-hypercyclic tuple for a subspace $M$ and $n=\left(n_{1}, n_{2}\right)$ is a multi-index, then must $T^{n}$ be an $M$ hypercyclic tuple?

The following example shows that the answer to the above question in general is not positive.

Example 3.6. Let $T_{1}=-2 I_{\mathbb{R}} \oplus I_{\mathbb{R}}$ and $T_{2}=\frac{1}{3} I_{\mathbb{R}} \oplus I_{\mathbb{R}}$. Then, $T=\left(T_{1}, T_{2}\right)$ is a subspace-hypercyclic tuple with respect to $M:=\mathbb{R} \oplus\{0\}$. But if we consider $n=(2,1)$, then $T^{n}=\left(T_{1}^{2}, T_{2}\right)$ is not subspace-hypercyclic with respect to $M:=\mathbb{R} \oplus\{0\}$. Since by Theorem 3.3, $\operatorname{cl}\left(\operatorname{orb}\left(T^{n},(1 \oplus 0)\right) \cap M\right)=[0,+\infty) \oplus\{0\}$ and $\operatorname{cl}\left(\operatorname{orb}\left(T^{n},(-1 \oplus 0)\right) \cap M\right)=(-\infty, 0] \oplus\{0\}$.

It is proved in [7] that compact operators are not subspace-hypercyclic.

Theorem 3.7. (see [7]) Let $T \in B(H)$, where $H$ is a Hilbert space. If $T$ is compact, then $T$ is not subspace-hypercyclic for any subspace.

In the following example we show that for a tuple $T=\left(T_{1}, T_{2}, \ldots, T_{n}\right), T$ may be a subspace-hypercyclic tuple while $T_{1}, T_{2}, \ldots, T_{n}$ are compact.

Example 3.8. Let $T=\left(-2 I_{\mathbb{R}} \oplus I_{\mathbb{R}}, \frac{1}{3} I_{\mathbb{R}} \oplus I_{\mathbb{R}}, T_{3}, \ldots, T_{n}\right)$ where $T_{3}, \ldots, T_{n}$ are compact operators on $\mathbb{R} \oplus \mathbb{R}$. By Theorem 3.3,

$$
\operatorname{cl}\left(\operatorname{orb}\left(T^{n},(1 \oplus 0)\right) \cap M\right)=\mathbb{R} \oplus\{0\} .
$$

So $T$ is a subspace-hypercyclic $n$-tuple with respect to $M:=\mathbb{R} \oplus\{0\}$. 


\section{References}

[1] S. I. Ansari, Hypercyclic and cyclic vectors, J. Funct. Anal., 128 (1998), 374-383, doi: 10.1006/jfan.1995.1036.

[2] F. Bayart, E. Matheron, Dynamics of linear operators, Cambridge University Press (2009).

[3] N. S. Feldman, Hypercyclic tuples of operators and somewhere dense orbits, J. Math. Appl., 346 (2008), 82-98, doi: 10.1016/j.jmaa.2008.04.027.

[4] G. Godefroy, J. H. Shapiro, Operators with dense, invariant, cyclic vector manifolds, J. Funct. Anal., 98(1991), 229-269, doi: 10.1016/0022-1236(91)90078-J.

[5] K.-G. Grosse-Erdmann, A.Peris Manguillot, Linear chaos, Springer (2011).

[6] C.M. Le, On subspace-hypercyclic operators, Proc. Amer. Math.Soc., B9( 2011), 28472852, doi: 10.1090/ S0002-9939-2011-10754-8.

[7] B. F. Madore, R. A. Martinez-Avendano, Subspace hypercyclicity, J. Math. Anal. Appl., 373(2011), 502-511, doi: 10.1016/j.jmaa.2010.07.049.

[8] M. Moosapoor, On point spectrum of subspace-hypercyclic operators, Int. J. Pure Appl. Math., 88(2013), 407-412, doi: 10.12732/ijpam.v88i3.8.

[9] M. Moosapoor, Irregular vectors and subspace-hypercyclic operators, Int. J. Pure Appl. Math., 86(2013), 1-6, doi: 10.12732/ijpam.v86i1.1.

[10] H. Rezaei, Notes on subspace-hypercyclic operators, J. Math. Anal. Appl., 397 (2013), 428-433, doi: 10.1016/j.jmaa.2012.08.002.

[11] R. Rolewicz, On orbits of elements, Studia Math., 32 (1969), 17-22.

[12] B.Yousefi, J. Izadi, Para-chaotic tuples of operators, The Australian Journal of Mathematical Analysis and Application. 9(1) (2012) 1-8. 